\title{
Correspondence
}

\section{Perioperative blood conservation strategies: weighing the medical evidence - I}

To the Editor:

We read, with interest, the recent editorial written by Drs. Karkouti and McCluskey. ${ }^{1}$ The crux of their editorial is that cardiac anesthesiologists should discontinue most blood conservation methods to avoid transfusions in heart surgery patients, until such methods have been proven safe and efficacious. However, the editorial leaves a number of unanswered questions; most importantly, is receiving blood, or blood component therapy, harmful? The authors suggest a causal relationship between the volume of transfusion and mortality. Work in this area has been based upon retrospective, propensity scored, database analysis which describes an association between transfusion and mortality from small to large volume transfusion. ${ }^{2-4}$ Possibly the nature of the surgical insult, with a requirement for large volume transfusion, is the major determinant for excess mortality, not the consequent transfusion. The findings of well-controlled trials, which examine simple methods to reduce transfusion requirements, can potentially be dismissed, arguing that they are not powered adequately to detect differences in mortality. However, it should be recognized that these studies were frequently not intended to evaluate mortality.

There is enormous variation in transfusion practice across centres performing the same type of cardiac operations. ${ }^{5}$ Many studies have evaluated methods that might reduce this variation in practice. Simple organizational changes and audit of practice may be just as effective in reducing transfusion rates as using mechanical or pharmacological methods. We have a safe blood supply; but this editorial largely ignores the wider issues around an increasingly scarce and expensive product. In addition, preventing low volume transfusions may be important to functional outcome after cardiac surgery. ${ }^{6}$

In the void left after the closure of the Blood conservation using antifibrinolytics: a randomized trial in a cardiac surgery population study, have Karkouti and McCluskey stopped using tranexamic acid in patients not involved in randomized clinical trials? Are they suggesting that units should stop using standard tests of coagulation in patients, as the value of these tests have not been validated in hemodiluted post-cardiopulmonary bypass coagulopathic patients?

The authors have produced a stimulating editorial. To do no harm, we must first correct the balance between transfusion and conservation approaches. This will require primary and database research into transfusion of blood, use of component therapy, and mechanical and pharmacological methods to reduce transfusion. At a time when aprotinin has been removed from our armamentarium, abandonment of a range of methods of transfusion avoidance may not be timely.

\section{Ravi Gill BM FRCA}

Mike Herbertson MBBS MRCP FRCA

Paul Diprose BM FRCSEd FRCA

Southampton University Hospital, Southampton, United Kingdom

E-mail: ravi@gill-taylor.com

Disclosure: All three authors have been in receipt of funding or honoraria from Novo Nordisk. Accepted for publication December 17, 2007.

\section{References}

1 Karkouti K, McCluskey SA. Perioperative blood conservation - The experts, the elephants, the clinicians and the gauntlet (Editorial). Can J Anesth 2007; 54: 861-7.

2 Engoren MC, Habib RH, Zacharias A, Schwann TA, Riordan CJ, Durham SJ. Effect of blood transfusion on long-term survival after cardiac operation. Ann Thorac Surg 2002; 74: 1180-6.

3 Koch CG, Li L, Duncan AI, et al. Morbidity and mortality risk associated with red blood cell and bloodcomponent transfusion in isolated coronary artery bypass grafting. Crit Care Med 2006; 34: 1608-16.

4 Karkouti K, Wijeysundera DN, Yau TM, et al. The independent association of massive blood loss with mortality in cardiac surgery. Transfusion 2004; 44 : 1453-62.

5 Stover EP, Siegel LC, Parks R, et al. Variability in transfusion practice for coronary artery bypass surgery persists despite national consensus guidelines: a 24-institution study. Institutions of the Multicenter Study of Perioperative Ischemia Research Group. 
Anesthesiology 1998; 88: 327-33.

6 Koch CG, Khandwala F, Li L, Estafanous FG, Loop FD,

Blackstone EH. Persistent effect of red cell transfusion on health-related quality of life after cardiac surgery.

Ann Thorac Surg 2006; 82: 13-20.

\section{Perioperative blood conservation strategies: weighing the medical evidence - II}

To the Editor:

In their recent editorial ${ }^{1}$ on the subject of perioperative blood conservation, Drs. Karkouti and McCluskey present a perspective which is at variance with some of the tenets of evidence-based medicine. ${ }^{1}$ As Drs. Goodenough and Shander have stated, "Blood management is most successful when multidisciplinary, proactive programs are in place so that these strategies can be individualized to specific patients". Evidence-based transfusion practice views a patient's blood as a valuable and a unique resource that should be managed appropriately. Allogeneic blood should only be used when there is evidence for potential benefit, when there are no safer alternatives, when a quality product is available, and after the risks have been appropriately considered. Patient-focused blood management is a multidisciplinary and a multimodal medical practice that starts with an accurate diagnosis of a patient's blood-related issues, through to the development of short and long-term management plans, with appropriate monitoring of outcomes.

On the basis of their editorial, ${ }^{1}$ it is uncertain how Drs. Karkouti and McCluskey would view the role of randomized controlled trials (RCTs) in confirming: the importance of pretransfusion compatibility testing; the avoidance of transfusion-transmitted viral diseases; the benefits of preoperative assessment for surgical bleeding and thromboembolic risk; and the benefits of correcting preoperative, reversible anemia in elective surgical cases. The recently published Austrian benchmark study on blood use in elective surgery ${ }^{3}$ demonstrates a high degree of interhospital variability in red cell transfusion rates for selected surgical procedures (e.g., for elective hip arthroplasty between $16 \%-85 \%$ of patients are transfused) that would be difficult to reconcile through a randomized controlled trial.

There is strong observational evidence indicating that perioperative, allogeneic red cell transfusion is an independent risk factor for increased morbidity and mortality. When concerns arise regarding the benefit and safety of a clinical intervention, especially where established alternatives are available, the precautionary principle should be applied. In transfusion medicine, this would mandate a non-transfusion default position. In this respect, it is ironic that blood transfusion is viewed differently from other therapies. Jehovah's Witness patients can safely undergo most surgical procedures, without allogeneic transfusion, with better clinical outcomes, especially in cardiac surgery, when compared with control matched, transfused patients. ${ }^{4}$

Drs. Karkouti and McCluskey imply that a numerical analysis frequentist approach to evidence (i.e., RCTs) is the only valid methodology to establishing causation where there is correlation, and that a sound understanding of pathophysiology and process decision analysis (Bayesian analysis) are of lesser validity. However, we should note that Dr. Archie Cochrane, of Cochrane Database fame, developed registries of observational data as the foundation for his epidemiological research. ${ }^{5}$ Even more importantly, Dr. Austin Bradford Hill proposed criteria for supporting causation from correlation when RCTs could not, or would never be done (e.g., smoking causing lung cancer). ${ }^{6}$ All medical therapies have associated risks and, recognizing that evidence for benefit is commonly lacking, RCTs have an important, but not exclusive, role in contributing to the establishment of best medical evidence. The recently identified safety concerns surrounding aprotinin and erythroid stimulating agents are concerning, and clearly need to be taken into account in assessing risk/benefit in modern perioperative transfusion medicine. Rather than being "elephants", aprotinin and erythroid stimulating agents are only two recently advocated pharmacological aspects of blood management. At the core of patient blood management is scientifically based clinical medicine dating back over 100 years.

The authors state that, for "the clinician", "The basic tenet of clinical medicine is, first, do no harm". They apply this dictum to two possible transfusion alternatives, but they do not acknowledge the vast observational and limited RCT data incriminating allogeneic transfusion as an independent risk factor for poorer clinical outcomes. The "experts" in patient blood management are not advocating the use of every blood conservation strategy in every patient, and are not striving for universal, bloodless medicine. However, the experts are advocating the practice of good clinical medicine, to minimize allogeneic blood transfusion and to conserve an altruistically donated resource that should be used for the benefit of the greatest number of patients. As for throwing down the "gauntlet", the patient blood management "gauntlet" has been at the authors' feet for many years. 